\title{
Design and analysis of SHE-assisted STT MTJ/CMOS logic gates
}

\author{
Prashanth Barla ${ }^{1}$. Vinod Kumar Joshi ${ }^{1}$ (1) . Somashekara Bhat ${ }^{1}$
}

Received: 12 May 2021 / Accepted: 4 August 2021 / Published online: 19 August 2021

(c) The Author(s) 2021

\begin{abstract}
We have investigated the spin-Hall effect (SHE)-assisted spin transfer torque (STT) switching mechanism in a three-terminal MTJ device developed using p-MTJ (perpendicular magnetic tunnel junction) and heavy metal materials of high atomic number, which possesses large spin-orbit interaction. Using p-MTJ schematic and complementary-metal-oxide-semiconductor (CMOS) logic, we have designed three basic hybrid logic-in-memory structure-based logic gates NOR/OR, NAND/ AND, and XNOR /XOR. Then the performances of these hybrid gates are evaluated and the results are compared with the conventional CMOS-based gates in terms of power, delay, power delay product, and device count. From the analysis, it is concluded that SHE-assisted STT MTJ/CMOS logic gates are nonvolatile, consume less power, and occupy a smaller die area as compared to conventional CMOS only logic gates.
\end{abstract}

Keywords Magnetic tunnel junction $\cdot$ Spin-hall effect $\cdot$ Spin transfer torque $\cdot$ Nonvolatile $\cdot$ Logic-in-memory

\section{Introduction}

Spintronics is one of the emerging areas that use the spin property of an electron in association with its charge [1,2]. It has an extra degree of freedom for computation. The spintronics research community has attracted much of the attention because already many efforts were made for various spintronics-based applications such as the development of nonvolatile (NV) memory [3-8], NV-logic implementation [9-18], and magnetic sensors for sensing magnetic field in pico-tesla range to the new growing technologies like neuromorphic computing and the brain-inspired computing, etc. [19-21]. In conventional electronics, which relies on the charge-based scalar quantity, the power (or heat) dissipation can be reduced up to a certain extent. The absence or presence of charge may correspond to logic " 0 " and logic " 1 ." In contrast, spin is a pseudovector quantity whose magnitude is fixed with variable polarization and denoted by $\hbar / 2$ (where $\hbar$ is the reduced Planck's constant). If an electron is placed in a magnetic field, various logic states can be achieved by varying the applied magnetic field. But, while designing the hybrid circuits, which

Vinod Kumar Joshi

vinodkumar.joshi@manipal.edu

1 Department of Electronics and Communication Engineering, Manipal Institute of Technology, Manipal Academy of Higher Education, Manipal 576104, India is a combination of conventional charge-based CMOS logic and spin-based spintronic devices, we need only two stable logic states, i.e., logic " 1 " and logic "0." In spintronics, a spinup and a spin-down electron are considered to be representing logic " 1 " and logic " 0 ," respectively, or vice versa. There are various spintronic devices that have been reported in the literature, such as spin-valve [22] magnetic tunnel junction (MTJ) [23], ferroelectric tunnel junction (FTJ) [24], domain wall $[13,25]$, skyrmion [26, 27], all-spin logic (ASL) devices [17, $18,28]$, etc. Among all the spintronic devices, we chose MTJ owing to its advantages such as simplicity, nonvolatility, large endurance, high density, fast reading capability, logic computation ability, 3D fabrication, and ease of integration with the existing CMOS technology, availability of models, and ease of manufacturing, etc. [1, 29, 30]. Alternatively, it consumes zero static power and has an instant ON-OFF feature. In the literature, there are various switching/writing techniques for MTJ device, such as spin-transfer torque (STT) [31-33], spin-orbit torque (SOT) [34-36], field-induced magnetic switching (FIMS) [37], and voltage-controlled magnetic anisotropy (VCMA) [38-40]. Considering the commercial aspect, the STT switching mechanism is more attractive among the rest [41-45]. Also, because of its simplicity, STT-MTJ can be easily incorporated into logic-in-memory (LIM) circuits. LIM is a new paradigm where computational capability is embedded into the memory, i.e., memory and processing of data/information is united in a single unit. In LIM, the memory which is 
involved in the processing of information is also nonvolatile in nature. LIM structure offers benefits over the conventional von-Neumann architecture, viz. lower power dissipation (both static and dynamic), scaling compatibility below sub-micron level (thereby increases the device density), immunity for the radiation effects, etc. [30, 46-48]. However, one of the key challenges for the LIM circuit is to improve the write speed and lowering the energy dissipation. The STT-MTJ device used in the LIM shows a large intrinsic incubation delay, which slows the write speed. Furthermore, incubation delay can contribute to circuit reliability issues. Because, to ensure the correct writing, we need a large write current flowing across the STT-MTJ. The flow of large write current causes a dielectric breakdown in the STT-MTJ over a period of time. This results in poor write endurance when we use only STT switching for MTJ devices [49]. In other words, it is challenging to maintain the critical current density $\left(J_{C}\right)$ while keeping high stability for the LIM structure. On the contrary, lowering $J_{C}$ can be achieved at the cost of lower stability. Also, lower $J_{C}$ causes read disturbance in STT-MTJ. There are several methods discussed in the literature to alleviate this trade-off [50-54]. But all the issues mentioned above can be addressed by spin-orbit torque (SOT) [55-57]. SOT is observed in a three-terminal device as shown in Fig. 1a. For STT, a spin-polarized current is needed to switch the magnetization of the free layer, while for SOT, this spin-polarized current is created by either the Rashba effect [58] or the spin-Hall effect (SHE) [35, 59]. The SOT induced by the Rashba effect is more subjected to debate [60]. Therefore, for the simplicity and availability of the MTJ model, we consider that SHE is the driving mechanism for SOT. However, the requirement of an additional external field hinders the development of SHE-MTJ-based circuits, which works purely on the SHE mechanism. As a

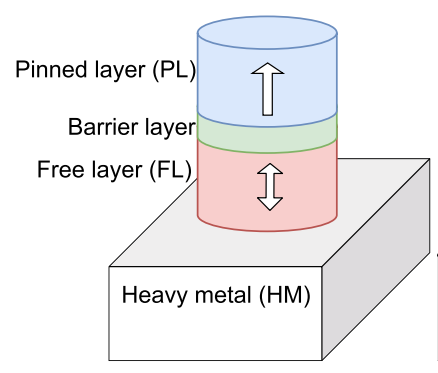

(a)

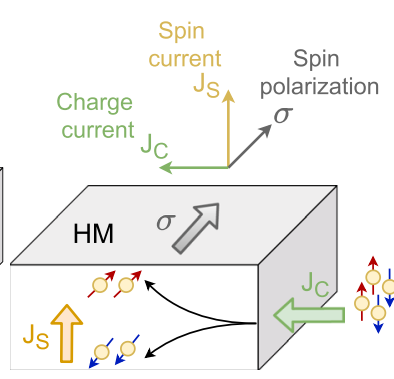

(b)
Fig. 1 a Schematic of SHE-assisted STT MTJ showing the pinned layer (PL) and free layer (FL) separated by a barrier layer. The FL is in contact with the heavy metal (HM). When the magnetic orientation of the FL and PL is pointing in the same direction, then the MTJ is in a parallel state $(\mathrm{P})$ with its resistance represented as $R_{P}$. On the contrary, when the magnetic orientation of the PL and FL is pointing in the opposite direction, then the MTJ is in the anti-parallel state (AP) and the resistance state is represented as $R_{A P}$. $\mathbf{b}$ Generation of pure spin current in the HM due to strong spin-orbit coupling illustrating the SHE effect [2] solution, SHE-assisted STT (SHE+STT) switching method was reported in the literature [61]. The SHE+STT switching technique has proved to be not only faster but also energy efficient. Using SHE+STT switching mechanism, various applications such as memory [62, 63], flip flop [64, 65], full adder $[66,67]$, and recently basic logic gates $[68,69]$ have already been developed. However the logic gates developed in Ref. [68] utilize in-plane-MTJ (i-MTJ) rather than perpendicularMTJ (p-MTJ). The i-MTJ suffers from disadvantages such as larger size, the difficulty for scaling, and low thermal stability, as compared to p-MTJ [2, 70, 71]. Further only AND and OR operations can be performed using the circuit proposed in Ref. [68]. Though Ref. [69] uses p-MTJ to develop the logic gate circuit, it can perform only NOT, AND/NAND, and OR/ NOR operations. Further to perform these operations, the circuit requires two passive capacitors to store the sensing voltage signals. But in VLSI design incorporation of passive devices is not preferred. In this paper, we have developed all the basic logic gates such as NOR/OR, NAND/AND, and XNOR/XOR using SHE-MTJ devices based on LIM structure, where the writing of the SHE-MTJ device is performed using the SHE+STT switching mechanism. Simulations are carried out to study the performance of these circuits in terms of an output response, power dissipation, power delay product (PDP), and the number of devices utilized in comparison with their CMOS counterparts; double pass-transistor logic-based clocked CMOS (DPTL-C ${ }^{2}$ MOS) logic gates. Further, we have also performed Monte Carlo (MC) simulations on these gates to study the power variations by incorporating process and mismatch variations in CMOS and extracted parameters of MTJ, which would arise during the fabrication process.

The paper is organized as follows: Sect. 2 presents the structure and working of SHE-MTJ device and hybrid LIM structure with MTJ/CMOS. In Sect. 3, we have explained the design and working of all the hybrid logic gates. Section 4 presents the performance evaluation of all the hybrid gates in terms of key performance indicators (KPI), power dissipation, delay, PDP, and device count. Comparison of these KPIs is compared with their CMOS counterparts. To study the power variations of various gates for process and mismatch (PM) variations, we have also conducted MC simulation. Finally, the paper is concluded in Sect. 5. Appendix representing the structure of (DPTL-C ${ }^{2} \mathrm{MOS}$ )-based logic gates has been also appended for convenience.

\section{Background}

SHE is considered as another phenomenon accountable for the SOT apart from Rashba effect [72]. Based on Mott scattering [73], Dyakonov and Perel predicted SHE in 1971 [34] and was revived by Hirsch in 1999 [35], the same was later demonstrated in $\mathrm{Pt}$ at room temperature by generating the 
substantial spin current [36]. Largely, SHE was believed to be arisen due to the skew scattering of s-electrons [35, 74], which is also known as an extrinsic mechanism. Materials with large SOI that also possess high atomic number such as $\mathrm{HM}$ convert the charge current to spin current exhibiting the SHE effect as shown in Fig. 1b. Here in HM, a flow of unpolarized electrons with charge current density $\left(\mathbf{J}_{C}\right)$ creates spin-polarized electrons with spin current density $\left(\mathbf{J}_{S}\right)$. Note that, $\mathbf{J}_{C}, \mathbf{J}_{S}$ and electron spin polarization $\boldsymbol{\sigma}$ are all perpendicular to each other, and the relationship between them is defined in Eq. 1 as,

$\mathbf{J}_{\mathbf{S}}=\theta_{\mathrm{SH}}\left(\boldsymbol{\sigma} \times \mathbf{J}_{\mathbf{C}}\right)$.

Where $\theta_{S H}$ is the spin-Hall angle characterizing the strength of SHE in HM. In the SHE-MTJ structure (Fig. 1a, the SHE generated in HM influences the direction of the free layer (FL). However, accurate switching cannot be guaranteed. This is because the direction of FL in p-MTJ and the electron spin in HM are not collinear. Accurate switching in p-MTJ can be achieved by the application of either an external magnetic field or a STT current. From the literature, it is clear that generation and maintenance of the external magnetic field is undesirable from a practical point of view due to its design complexity, reduced sensitivity, and thermal stability [2]. Hence, using STT current in conjunction with SHE is a more suitable option for precise switching in p-MTJ [61]. This is called as SHE+STT switching in our manuscript. Figure 2 shows the switching of a three-terminal p-MTJ device structure with SHE+STT currents.

Switching the p-MTJ from AP to P (Fig. 2a) is explained as follows when the SHE current $J_{S H E}$ flows in Y-direction (from T3 to T2), and spin accumulation is created at the FL-HM interface. This exerts a SOT onto the FL to tilt its magnetic orientation from $-\mathrm{Z}$-direction to the $\mathrm{X}$-Y plane. At the same time, the STT current $\left(J_{S T T}\right)$ flowing in the -Z-direction (from T1 to T2) exerts STT and switches the orientation of FL from XY-plane to Z-direction. On the contrary, to switch p-MTJ from P to AP (Fig. 2b), the direction of $J_{S T T}$ is reversed (from $-\mathrm{Z}$ to $\mathrm{Z}$ ).

\subsection{Logic-in-memory structure}

Initially, Kautz put forth the idea of LIM in 1969 [75], and later in a year, i.e., in 1970, Stone presented an approach for LIM computer [76]. But later it did not attract much attention. However recently, due to the slowing trend of transistor scaling, advancements in thin films, and the emergence of spintronics devices, LIM concept is drawing the notice of both academia and industry. LIM can offer solutions for the problems of increased leakage current due to scaling and considerable delay in long interconnects by positioning the NV memory section above the CMOS logic [46]. MTJs

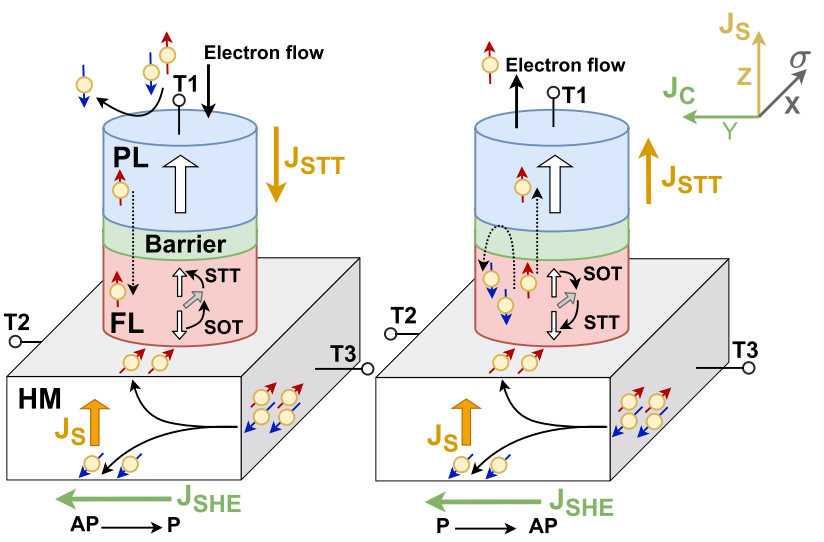

Fig. 2 SHE-assisted STT MTJ switching mechanism in a p-MTJ device. a AP to P switching of the MTJ, when $J_{S H E}$ and $J_{S T T}$ flow along $\mathrm{Y}$ and $-\mathrm{Z}$ directions, respectively. $\mathbf{b}$ For $\mathrm{P}$ to AP switching of the MTJ, the direction of $J_{S H E}$ is the same while the direction of $J_{S T T}$ has been reversed [2]

provide nonvolatility to the structure. As a result, the power supply to the idle block can be turned off without losing the information stored in the MTJ. When the power is restored this information is readily available for processing without the need for the restore operation. Hence, the static power dissipation is almost zero in the standby mode. Therefore this approach is convenient for the "instant-on" and "normally-off" system [77]. To add to the above advantage, the 3D stacking feasibility of MTJ on top of CMOS not only reduces the latency but also increases the integration density in the LIM structure. A schematic of the LIM structure with three major components is illustrated in Fig. 3 as,

1. Sense amplifier/Read circuit: It produces Out and $\overline{\mathrm{Out}}$ based on the differential current $\left(I_{L}\right.$ and $\left.I_{R}\right)$ flowing through the logic network.

2. Write circuit: This is used to write the information (or change the states of MTJ) in pair of MTJ. Based on the various writing mechanisms, there will be a change in the write circuitry.

3. Logic network: Various logical operations can be performed based on the design of the nMOS logic tree and information stored in the MTJ pair.

\section{Hybrid MTJ/CMOS LIM-based logic gates}

\subsection{Selection and working of MTJ reading circuit}

We have adopted the reading circuit proposed by [78], which is a modified version of the conventional pre-charge sense amplifier (PCSA) discussed in Ref. [79]. We address the PCSA from Ref. [79] as PCSA1 and from Ref. [78] as PCSA2. PCSA2 is superior to PCSA1 in terms of speed and 


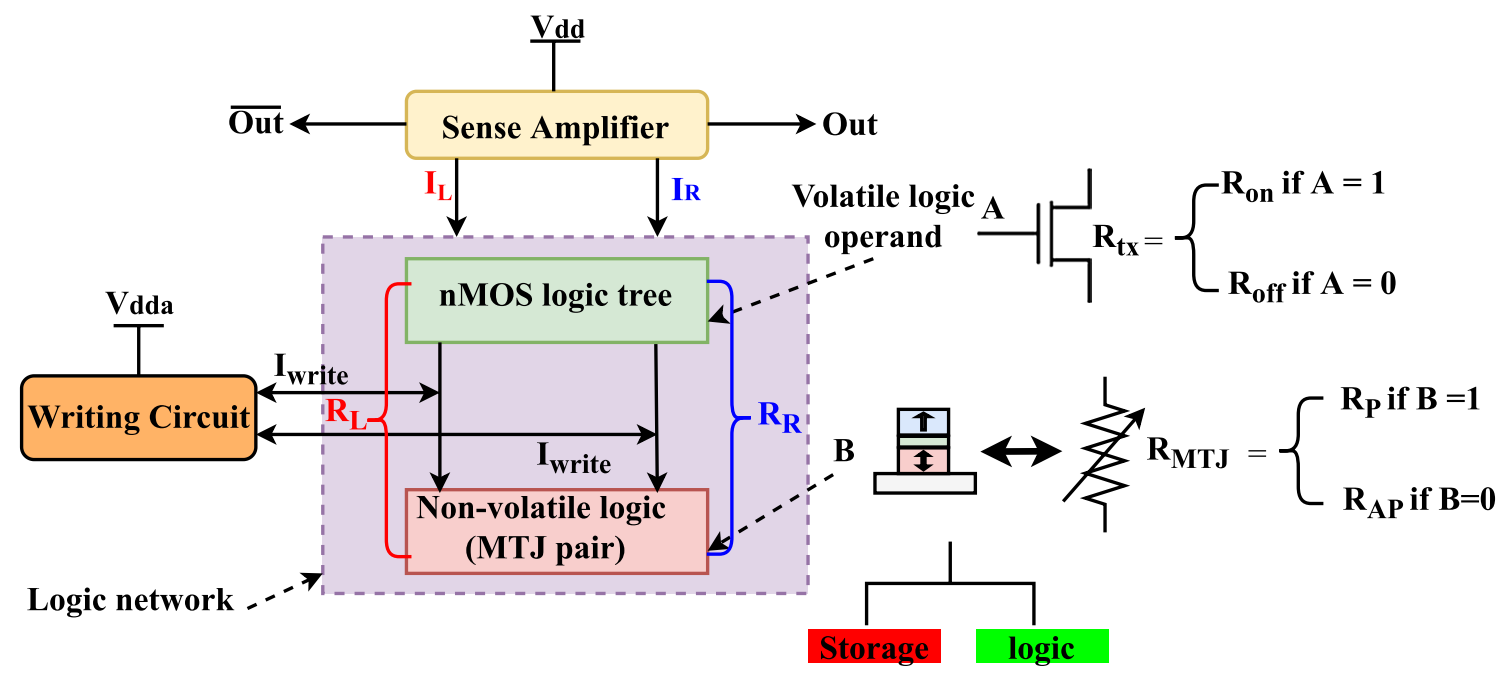

Fig. 3 Schematic of a hybrid MTJ/CMOS LIM structure [1]. Here, $I_{\text {write }}$ is the bi-directional write current generated by the writing circuit, $R_{L}$ and $R_{R}$ are the left and right branch total resistances of the nMOS logic tree and MTJs, respectively. A and B are the two logic operands represented by the nMOS and three terminal MTJ

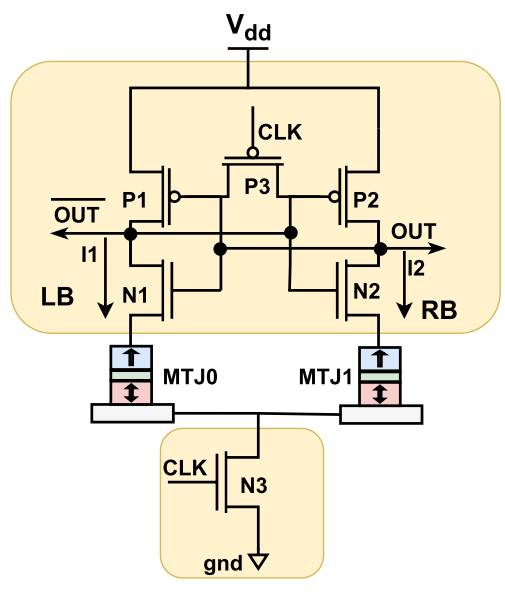

Fig. 4 Schematic of the PCSA2 circuit which reads the bit stored in a pair of MTJs [78]

power/energy consumption and the schematic of PCSA2 is shown in Fig. 4. The PCSA2 circuit works in two phases: the pre-charge phase and the evaluation phase. In pre-charge phase both outputs, OUT and $\overline{\text { OUT }}$ are high. During the evaluation phase, the values stored in the MTJs are read and reflected at the output nodes. When the bit " 0 " is stored, the MTJ pair (MTJ0-MTJ1) is in AP-P configuration; on the contrary, when bit " 1 " is stored, the MTJ pair is configured as P-AP. The detailed working of the reading circuit is explained by considering bit " 0 " is stored in the MTJ pair as follows: Since the output of the hybrid circuits is complementary in nature, initially we consider OUT is at logic " 0 " and $\overline{\mathrm{OUT}}$ is at logic " $1 . "$
1. In the pre-charge phase: Clock $(\mathrm{CLK})=$ " 0 " transistors $\mathrm{P} 3$ is ON and N3 is OFF. The OUT and $\overline{\mathrm{OUT}}$ node voltages will be shared through $\mathrm{P} 3$ and both OUT and $\overline{\mathrm{OUT}}$ will be pre-charged to Vdd-Vth (In PCSA1 [79], it was Vdd).

2. In the evaluation phase: $\mathrm{CLK}=$ " 1 ," transistor $\mathrm{N} 3$ is turned ON, and it offers a discharging path for OUT as well as $\overline{\mathrm{OUT}}$ nodes to gnd. The current I1 and I2 in the left branch (LB) and right branch (RB), respectively, start to flow towards gnd at a different rate. This is due to the resistance variation between MTJ0 and MTJ1. As MTJ0 and MTJ1 are at $R_{A P}$ and $R_{P}$, respectively, I2 discharges faster than I1. It causes, OUT node to be pulled down the threshold voltage of P1, turning it ON. So the node $\overline{\text { OUT }}$ is raised to logic " 1. ." On the contrary, OUT keeps discharging to gnd and reaches logic " 0 ." So sensing/reading bit " 0 " takes place from MTJ0-MTJ1. Similarly, we can understand reading bit "1" from the MTJ pair when it is configured as P-AP.

PCSA2 produces a quicker output response than PCSA1 because, in the pre-charge phase of PCSA2 both the outputs, i.e., OUT and $\overline{\mathrm{OUT}}$ are pre-charged to $\mathrm{Vdd}-\mathrm{Vth}$, whereas in PCSA1, OUT and $\overline{\text { OUT }}$ are pre-charged to Vdd. In the evaluation phase, when one of the outputs of PCSA2 discharges to gnd from Vdd-Vth, it takes lesser time as compared to PCSA1, where Vdd needs to be discharged to gnd. Consequently, the lower voltage (Vdd-Vth) in PCSA2 dissipates lower power than PCSA1. Further, PCSA2 works with one PMOS lesser than PCSA1, which helps to reduce the transistor count. 


\subsection{SHE+STT MTJ writing circuit for LIM structure}

Figure 5 shows the writing circuit for SHE-MTJs with the SHE+STT switching mechanism. The writing driver is divided into two parts: control circuitry and writing core. It has three inputs, viz. Data, EnW, and EnSHE. Table 1 shows the different combinations of the input signals, control signals, and the corresponding status of the MTJ pair. When EnW is at logic " 0 ," the writing core is disabled by the intermediate signals (STTP, STTN, SHEP, SHEN = "1010") of the control circuit, and no writing takes place. Information will be written only when EnW = "1." Consider writing bit "1" into the MTJ pair, for which EnW, EnSHE, and Data are set as "111," respectively. So the intermediate signals STTP, STTN, SHEP, SHEN will be at "1101," respectively. This will turn ON transistors MP1, MP2, MP3,
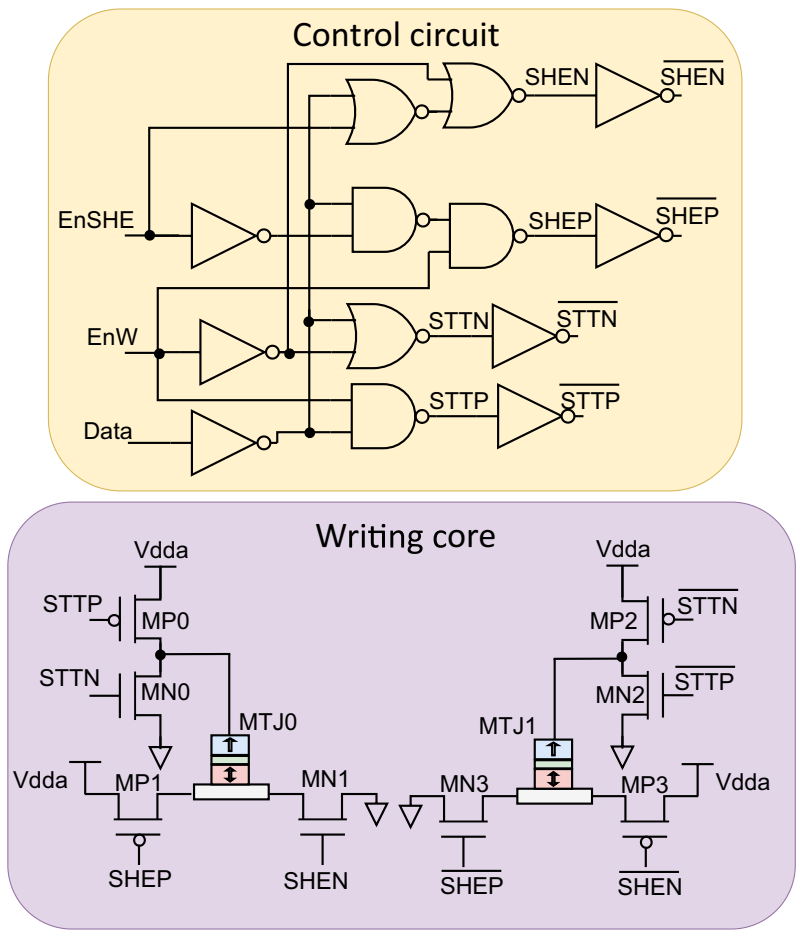

Fig. 5 Schematic of the MTJ write circuit working with the SHE+STT writing mechanism [64]
MN0, MN1, MN3. Two types of current are flowing in the circuit, i.e., SHE and STT current. SHE current path is; Vdda-MP1-MTJ0-MN1-gnd and Vdda-MP3-MTJ1-MN3gnd. STT current path is; Vdda-MP2-MTJ1-MN3-gnd and Vdda-MP1-MTJ0-MN0-gnd. At this point, the MTJ pair is in a metastable state. After a brief period, EnSHE is asserted to "0" , making STTP, STTN, SHEP, SHEN = "1100." This will turn OFF the transistors MP3 and MN1 which stops the flow of SHE current in the circuit. But STT current flows continuously to change the MTJ0-MTJ1 configuration to P-AP, respectively. Similarly, Data " 0 " can be written into the MTJ pair by suitably changing the input signals as shown in Table 1.

\subsection{Hybrid MTJ/CMOS LIM-based logic gates}

Figure 6a-c shows the circuit for NOR/OR, NAND/AND and XNOR/XOR gates, respectively, and Table 2 shows corresponding truth table. These hybrid circuits operate in the pre-charge and evaluation phase. In the pre-charge phase inputs are applied and in the evaluation phase output and its complement are obtained. The working of the NOR/OR gate in its evaluation phase is as follows. Whenever input $\mathrm{A}=$ " 1 " , N6 in the right branch (RB) is open and N5 in the left branch (LB) is ON. The total RB resistance for the discharge current (I2) is the sum of OFF-transistor (N6) resistance $\left(R_{\text {OFF }}\right)$ and MTJ1 resistance $\left(R_{P} / R_{A P}\right)$. Similarly, total LB resistance for the discharge current (I1) is the sum of ON-transistor (N5) resistance $\left(R_{O N}\right)$ and MTJ0 resistance $\left(R_{P} / R_{A P}\right)$. In this case, since transistor N6 is OFF, RB resistance is always greater than the LB resistance. The state of the MTJ does not affect the output, as $R_{O F F}$ is always greater than either $R_{O N}+R_{A P}$ or $R_{O N}+R_{P}\left(R_{O F F}>\left(R_{O N}+R_{A P}\right)\right.$ or $\left.R_{O F F}>\left(R_{O N}+R_{P}\right)\right)$. Therefore OR output node is raised to Vdd, and the NOR node discharge to gnd; hence, it is at logic " 0 ," whereas when the $\mathrm{A}=$ " 0 ," N4 and N6 in LB and RB are ON, due to that NOR and OR outputs have a discharging path to gnd. In this condition, the bit stored in B decides the value of output nodes. If $\mathrm{B}=$ " 0 ," the state of the MTJ0-MTJ1 is in AP-P configuration. So the total resistance observed for currents I1 and I2 is $R_{O N}+R_{A P}$ and $R_{O N}+R_{P}$, respectively. Thus the LB resistance is comparatively greater than RB. This results in the NOR
Table 1 Input, intermediate signals and the corresponding MTJ states during SHE+STT writing

\begin{tabular}{|c|c|c|c|c|c|c|c|c|}
\hline \multicolumn{3}{|c|}{ Input signals } & \multicolumn{4}{|c|}{ Intermediate signals } & \multicolumn{2}{|l|}{ MTJ status } \\
\hline EnW & EnSHE & Data & STTP & STTN & SHEP & SHEN & MTJ0 & MTJ1 \\
\hline 0 & $X$ & $X$ & 1 & 0 & 1 & 0 & $X$ & $X$ \\
\hline 1 & 1 & 1 & 1 & 1 & 0 & 1 & Metastable & \\
\hline 1 & 0 & 1 & 1 & 1 & 0 & 0 & $\mathrm{P}$ & $\mathrm{AP}$ \\
\hline 1 & 1 & 0 & 0 & 0 & 0 & 1 & Metastable & \\
\hline 1 & 0 & 0 & 0 & 0 & 1 & 1 & AP & $\mathrm{P}$ \\
\hline
\end{tabular}

$\mathrm{X}$ represents don't care condition 


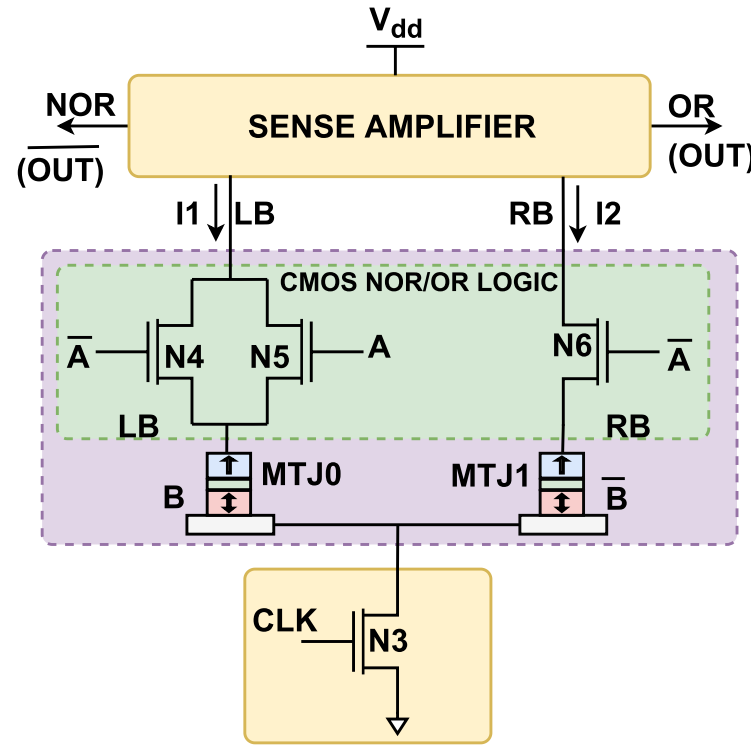

(a)

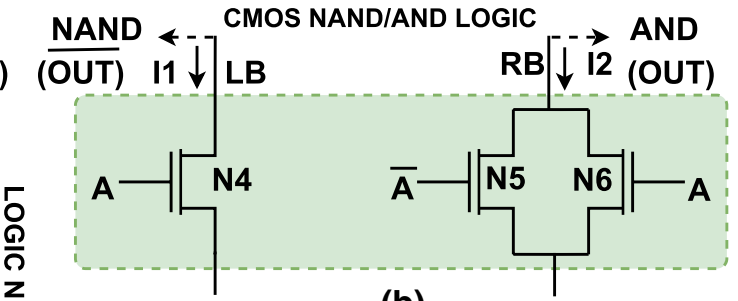

(b)

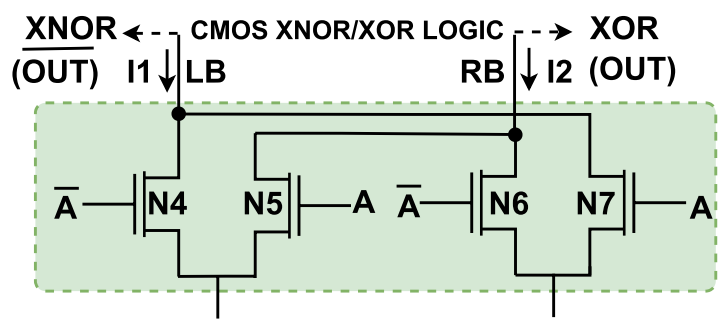

(c)

Fig. 6 Hybrid MTJ/CMOS a NOR/OR b NAND/AND and $\mathbf{c}$ XNOR/XOR logic gates based on LIM

Table 2 Truth table for various logic gates along with the corresponding path resistance for the branch current in logic network

\begin{tabular}{|c|c|c|c|c|c|c|c|}
\hline \multirow[t]{2}{*}{ Gate type } & \multicolumn{2}{|c|}{ Inputs } & \multirow{2}{*}{$\begin{array}{l}\text { LB resistance } \\
\text { For } \mathrm{I} 1\left(R_{L B}\right)\end{array}$} & \multirow{2}{*}{$\begin{array}{l}\mathrm{RB} \text { resistance } \\
\text { for } \mathrm{I} 2\left(R_{R B}\right)\end{array}$} & \multirow{2}{*}{$\begin{array}{l}\text { Resistance } \\
\text { Comparison }\end{array}$} & \multirow[t]{2}{*}{ OUT } & \multirow[t]{2}{*}{$\overline{\text { OUT }}$} \\
\hline & A & B & & & & & \\
\hline \multirow[t]{4}{*}{ NOR/OR } & 0 & 0 & $R_{O N}+R_{A P}$ & $R_{O N}+R_{P}$ & $R_{L B}>R_{R B}$ & 0 & 1 \\
\hline & 0 & 1 & $R_{O N}+R_{P}$ & $R_{O N}+R_{A P}$ & $R_{L B}<R_{R B}$ & 1 & 0 \\
\hline & 1 & 0 & $R_{O N}+R_{A P}$ & $R_{\text {OFF }}+R_{P}$ & $R_{L B}<R_{R B}$ & 1 & 0 \\
\hline & 1 & 1 & $R_{O N}+R_{P}$ & $R_{O F F}+R_{A P}$ & $R_{L B}<R_{R B}$ & 1 & 0 \\
\hline \multirow[t]{4}{*}{ NAND/AND } & 0 & 0 & $R_{\text {OFF }}+R_{A P}$ & $R_{O N}+R_{P}$ & $R_{L B}>R_{R B}$ & 0 & 1 \\
\hline & 0 & 1 & $R_{\text {OFF }}+R_{P}$ & $R_{O N}+R_{A P}$ & $R_{L B}>R_{R B}$ & 0 & 1 \\
\hline & 1 & 0 & $R_{O N}+R_{A P}$ & $R_{O N}+R_{P}$ & $R_{L B}>R_{R B}$ & 0 & 1 \\
\hline & 1 & 1 & $R_{O N}+R_{P}$ & $R_{O N}+R_{A P}$ & $R_{L B}<R_{R B}$ & 1 & 0 \\
\hline \multirow[t]{4}{*}{ XNOR/XOR } & 0 & 0 & $R_{O N}+R_{A P}$ & $R_{O N}+R_{P}$ & $R_{L B}>R_{R B}$ & 0 & 1 \\
\hline & 0 & 1 & $R_{O N}+R_{P}$ & $R_{O N}+R_{A P}$ & $R_{L B}<R_{R B}$ & 1 & 0 \\
\hline & 1 & 0 & $R_{O N}+R_{P}$ & $R_{O N}+R_{A P}$ & $R_{L B}<R_{R B}$ & 1 & 0 \\
\hline & 1 & 1 & $R_{O N}+R_{A P}$ & $R_{O N}+R_{P}$ & $R_{L B}>R_{R B}$ & 0 & 1 \\
\hline
\end{tabular}

$R_{O N}:$ NMOS, ON resistance. $R_{O F F}:$ NMOS, OFF resistance.

$R_{P}$ : P state resistance of MTJ. $R_{A P}$ : AP state resistance of MTJ node being raised to logic " 1 "; correspondingly, OR node will be discharged to gnd. In another instance, when $\mathrm{A}=$ " 0 " and $\mathrm{B}=$ "1," MTJ pair is at P-AP configuration. Resistances of $\mathrm{LB}$ and $\mathrm{RB}$ are at $R_{O N}+R_{P}$ and $R_{O N}+R_{A P}$ for the currents I1 and I2, respectively. Now the LB resistance is lower than RB. Hence, OR output node raises to logic " 1 " and the NOR output node discharges to gnd, producing logic " 0 ." Similarly working of NAND/AND and XNOR/XOR gates can be understood.

\section{Simulation results and discussion}

We have performed electrical simulations in $45 \mathrm{~nm}$ CMOS generic process design kit using the Cadence tool (IC 6.1.764b.500.19) with default transistor parameters, $\mathrm{L}=45 \mathrm{~nm}$ and $\mathrm{W}=120 \mathrm{~nm}$. The SHE-MTJ electrical model developed by Ref. [60] is used in our simulation work. This model is developed using the Verilog-A language. Table 3 shows the 
MTJ parameters set during the simulation with the SHEMTJ model, whereas the other parameters are retained as default values as mentioned in Ref. [60]. We have set a supply voltage of $\mathrm{Vdda}=1.2 \mathrm{~V}$ for MTJ writing purpose, and $\mathrm{Vdd}=1 \mathrm{~V}$ for MTJ reading operations and DPTL-C ${ }^{2} \mathrm{MOS}$ logic. With Vdda, we ensure that there is no area overhead caused by the writing core transistors; meanwhile, the MTJ write current is larger than the critical current so that switching of the MTJ can be accomplished with $100 \%$ probability.

\subsection{Model verification of SHE-assisted STT MTJ model}

In this section, we have performed model verification of SHE-MTJ and studied its switching behavior. Figure 7a, b show the waveform of SHE-MTJ model for three particular cases with SHE+STT, STT only (SHE current is zero), and SHE only (STT current is zero) simulation for AP to $\mathrm{P}$ and $\mathrm{P}$ to AP switching condition, respectively [60]. As seen in Fig. 7a, the switching time for AP to P switching with SHE+STT mechanism is at T1, whereas with STT only mechanism, it is at T2. On the contrary, with SHE only, the switching of MTJ does not take place. Similarly, as seen in Fig. 7b, the switching time for P to AP switching with SHE+STT mechanism is at T3, whereas it is at T4 with STT only mechanism. In this case also, with SHE only, switching does not take place. With SHE+STT switching mechanism, we have obtained T2-T1 and T4-T3, as the improvement in switching time for AP to P and P to AP switching, respectively, as compared to STT only switching mechanism. This is due to the elimination of incubation delay with the SHE+STT switching mechanism.

Figure 8 shows the analysis of varying SHE pulse width, i.e., from 0 to $355 \mathrm{ps}$, on the switching process of the SHE+STT switching mechanism. It is observed that when

Table 3 SHE-MTJ parameters set during the simulation [60]

\begin{tabular}{lll}
\hline Parameter & Description & Value \\
\hline$t_{s l}$ & Free layer thickness & $0.7 \mathrm{~nm}$ \\
$t_{o x}$ & MgO barrier thickness & $0.85 \mathrm{~nm}$ \\
TMR & TMR ratio under zero bias voltage & $200 \%$ \\
Shape & MTJ Surface shape & Circle \\
a & MTJ Surface length & $32 \mathrm{~nm}$ \\
$\mathrm{~b}$ & MTJ Surface width & $32 \mathrm{~nm}$ \\
$\mathrm{r}$ & MTJ Surface radius & $16 \mathrm{~nm}$ \\
$\mathrm{w}$ & Heavy-metal width & $40 \mathrm{~nm}$ \\
$\mathrm{~d}$ & Heavy-metal thickness & $3 \mathrm{~nm}$ \\
1 & Heavy-metal length & $60 \mathrm{~nm}$ \\
$\sigma_{T M R}$ & Standard deviation of TMR & $3 \% \mathrm{of} T M R$ \\
$\sigma_{t_{s l}}$ & Standard deviation of $t_{s l}$ & $3 \% \mathrm{of} t_{s l}$ \\
$\sigma_{t_{o x}}$ & Standard deviation of $t_{o x}$ & $3 \%$ of $t_{o x}$ \\
\hline
\end{tabular}

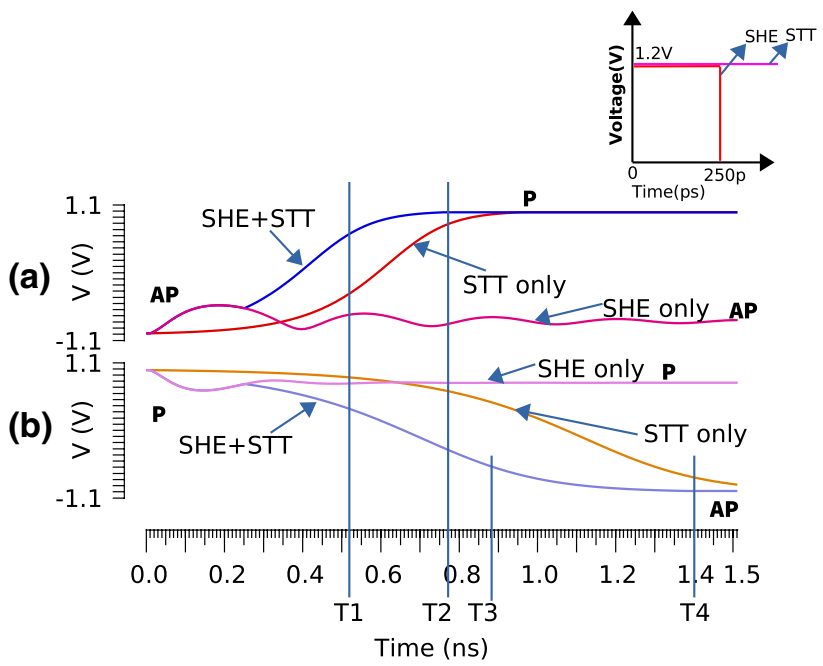

Fig. 7 Simulated waveform of a single-cell SHE-MTJ for a AP to P and $\mathbf{b} \mathrm{P}$ to AP switching. While obtaining the switching times (T1 to T4), a supply of $1.2 \mathrm{~V}$, and SHE pulse width of $250 \mathrm{~ns}$ are applied. Other MTJ parameters are set according to Table 3. When the signal crosses $90 \%$ of its maximum value we consider that the MTJ switching is complete

SHE pulse width is zero, STT only switching takes place. The switching time is minimum when SHE pulse width is set to 100 ps. Figure 9 shows the simulated waveform for the writing circuit which works on the SHE+STT switching mechanism. Initially bit " 1 " is stored in the MTJ pair. At time T1, EnW and EnSHE enable the writing of bit " $0 . "$ At time T2 bit "1" is written. At time T3, bit "1" is being

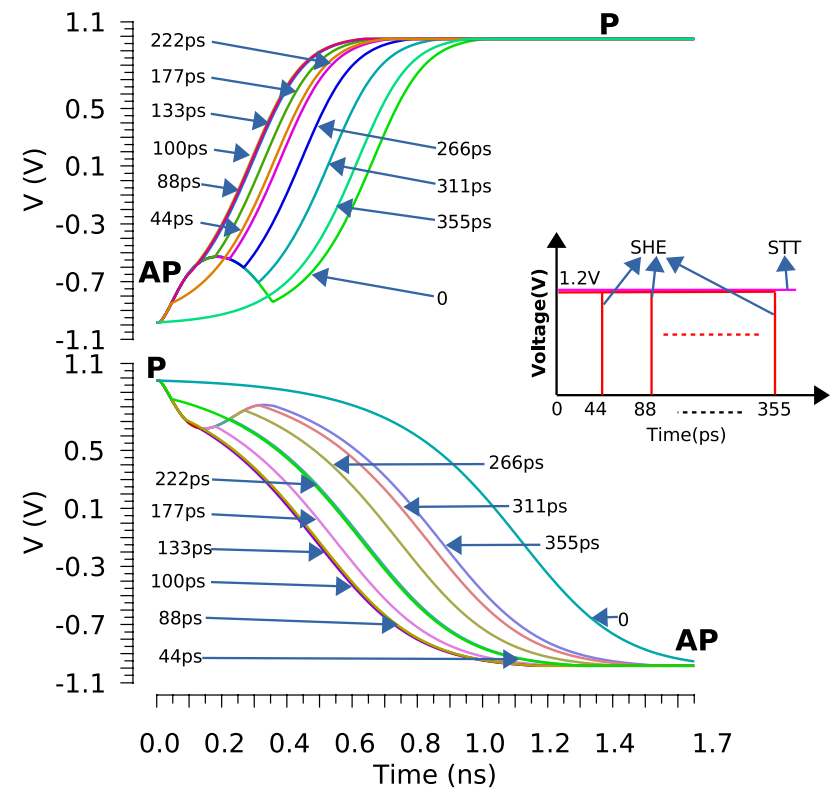

Fig. 8 Simulated waveform for a single-cell SHE-MTJ with the SHE pulse width varying from 0 to $355 \mathrm{ps}$ 


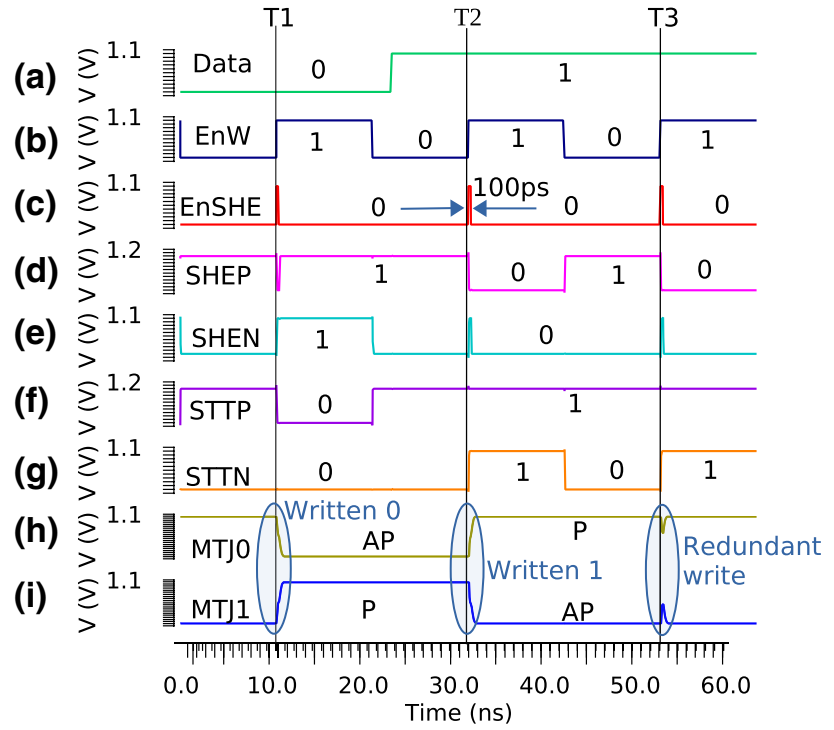

Fig. 9 Simulated waveform for the SHE-MTJ model with SHE+STT writing mechanism

Table 4 Performance comparison between SHE+STT and STT only writing

\begin{tabular}{|c|c|c|c|c|}
\hline \multicolumn{2}{|l|}{ Particulars } & \multirow{2}{*}{$\begin{array}{l}\text { SHE+STT } \\
819.7 \mathrm{f}\end{array}$} & \multirow{2}{*}{$\begin{array}{l}\text { STT only } \\
812.4 \mathrm{f}\end{array}$} & \multirow{2}{*}{$\begin{array}{l}\text { No. of devices } \\
\mathrm{MOS}+2 \mathrm{MTJ}\end{array}$} \\
\hline & Write 0 & & & \\
\hline Writing core & Write 1 & $819.3 \mathrm{f}$ & $812.3 \mathrm{f}$ & \\
\hline \multirow[t]{4}{*}{ Energy/bit (J) } & Redundant & & & \\
\hline & Write & $819.3 f$ & $812.2 \mathrm{f}$ & \\
\hline & Average $^{\mathrm{x}}$ & $819.4 f$ & $812.3 \mathrm{f}$ & \\
\hline & Write 0 & $3.23 \mathrm{f}$ & $817.9 \mathrm{a}$ & 38MOS \\
\hline Control circuit & Write 1 & $2.74 \mathrm{f}$ & $986.9 \mathrm{a}$ & \\
\hline \multirow[t]{3}{*}{ Energy/bit (J) } & Redundant & & & \\
\hline & Write & $2.67 f$ & $822.4 a$ & \\
\hline & Average $^{y}$ & $2.88 \mathrm{f}$ & $875.7 \mathrm{a}$ & \\
\hline \multicolumn{2}{|l|}{ Total $^{\mathrm{x}+\mathrm{y}}$} & $822.28 \mathrm{fJ}$ & $813.17 \mathrm{fJ}$ & $46 \mathrm{MOS}+2 \mathrm{MTJ}$ \\
\hline \multicolumn{2}{|c|}{ Worst-case delay (ps) } & 386.82 & 900 & \\
\hline
\end{tabular}

${ }^{\mathrm{x}, \mathrm{y}}$ Represent the average energy/bit for writing core and control circuit respectively

rewritten into the MTJ pair, which is known as redundant write. Table 4 shows the comparison in terms of energy consumption, device count, and worst-case switching delay for SHE+STT and STT only writing process. Here we can notice that the average energy/bit consumption for STT only switching is $813.17 \mathrm{fJ}$, whereas, with SHE+STT switching, it is $822.28 \mathrm{fJ}$. There is a marginal decrease in the average energy/bit (i.e., 9.11fJ) with STT only as compared to SHE+STT switching. However, the worst-case delay for SHE+STT switching is 386.82ps, whereas for the STT only switching it is 900ps. With SHE+STT switching, the worst-case delay is decreased by $57.02 \%$. Moreover, due to stochasticity and incubation delay associated with STT only switching, there is no guaranteed $100 \%$ switching in STT only. Hence, SHE+STT switching is the better option over STT only.

\subsection{Performance analysis of hybrid MTJ/CMOS logic gates}

We address the hybrid NOR/OR, NAND/AND, and XNOR/ XOR logic gates with PCSA1 as HG1 and hybrid logic gates with PCSA2 as HG2, whereas its corresponding CMOS counterparts are developed using DPTL- ${ }^{2}$ MOS logic and we address them as DPTL- ${ }^{2}$ MOS logic gates. The comparison between hybrid and DPTL-C ${ }^{2}$ MOS-based NOR/OR, NAND/AND, and XOR/XNOR gate operations in terms of power, delay, PDP, and device count is presented in Table 5 . The static power dissipation of HG1 and HG2 gates is considered to be zero in comparison with their CMOS counterparts because, in the hybrid logic gate, we store the input B in the nonvolatile MTJ pair, due to which we can turn off the power supply in the standby mode without losing the information. In the active mode, the stored information is readily available for computation without the need for a write/restoration process. However, there is steady-state power dissipation observed for the hybrid logic gates which are shown in Table 5.

The dynamic power dissipation in HG2 is least as compared to its corresponding HG1 and DPTL-C ${ }^{2}$ MOS logic gates. The dynamic power dissipation for NOR/OR, NAND/ AND, and XNOR /XOR logic gates in HG2 is less than HG1 by $51.7 \%, 55.11 \%$ and $53.23 \%$, respectively, and it is less than its DPTL-C ${ }^{2}$ MOS logic gates by $66.11 \%, 66.23 \%$ and $62.03 \%$, respectively. Similarly, the total power dissipation of NOR/OR, NAND/AND, and XNOR /XOR logic gates in HG2 is less than HG1 by $23.42 \%, 22.31 \%$, and $27.84 \%$ and it is less than DPTL-C ${ }^{2}$ MOS logic gates by $24.85 \%, 27.84 \%$ and $29.02 \%$, respectively. Figure $10 \mathrm{a}$, b shows a bar chart plot for the dynamic as well as total power dissipation comparison between HG1, HG2 and DPTL-C ${ }^{2}$ MOS-based NOR/ OR, NAND/AND and XNOR/XOR logic gates, respectively.

The delay depends upon the quality of the sense amplifier used in hybrid logic gates. In HG2 with PCSA2, during the pre-charge phase both output and its complement are at voltage Vdd-Vth, whereas for HG1 with PCSA1, output and its complement are at voltage Vdd. Hence during the evaluation phase, HG2 with PCSA2 discharges one of its output to gnd quicker than its corresponding HG1 with PCSA1 counterpart, producing a faster output response. But the DPTL$\mathrm{C}^{2} \mathrm{MOS}$ logic gates have lesser delay than hybrid gates. This is because in DPTL-C ${ }^{2}$ MOS logic operates based on clocked CMOS pass transistor logic, whereas the hybrid gates use current-controlled sense amplifier. In the evaluation phase, 
Table 5 Comparison between various logic gates with different read circuits in terms of power dissipation and device count at an operating frequency of $500 \mathrm{MHz}$

\begin{tabular}{|c|c|c|c|c|c|c|c|c|c|}
\hline Gate & HG1 & HG2 & $\begin{array}{l}\text { DPTL- } \\
\mathrm{C}^{2} \mathrm{MOS}\end{array}$ & HG1 & HG2 & $\begin{array}{l}\text { DPTL- } \\
\mathrm{C}^{2} \mathrm{MOS}\end{array}$ & HG1 & HG2 & DPTL-C ${ }^{2}$ MOS \\
\hline type & NOR/OR & NOR/OR & NOR/OR & NAND/AND & NAND/AND & NAND/AND & XNOR/XOR & XNOR/XOR & XNOR/XOR \\
\hline $\begin{array}{l}\text { Read } \\
\text { type }\end{array}$ & PCSA1 & PCSA2 & $\begin{array}{l}\text { DPTL- } \\
\mathrm{C}^{2} \mathrm{MOS}\end{array}$ & PCSA1 & PCSA2 & $\begin{array}{l}\text { DPTL- } \\
\mathrm{C}^{2} \mathrm{MOS}\end{array}$ & PCSA1 & PCSA2 & DPTL-C ${ }^{2} \mathrm{MOS}$ \\
\hline $\begin{array}{l}\text { Static } \\
\text { Power }(\mathrm{nW})\end{array}$ & $\begin{array}{l}0^{\mathrm{a}} \\
\left(321.15^{\mathrm{b}}\right)\end{array}$ & $\begin{array}{l}0^{\mathrm{a}} \\
\left(312.35^{\mathrm{b}}\right)\end{array}$ & $231.8^{\mathrm{c}}$ & $\begin{array}{l}0^{\mathrm{a}} \\
\left(274.6^{\mathrm{b}}\right)\end{array}$ & $\begin{array}{l}0^{\mathrm{a}} \\
\left(285.35^{\mathrm{b}}\right)\end{array}$ & $240.2^{\mathrm{c}}$ & $\begin{array}{l}0^{\mathrm{a}} \\
\left(324.4^{\mathrm{b}}\right)\end{array}$ & $\begin{array}{l}0^{\mathrm{a}} \\
\left(311.1^{\mathrm{b}}\right)\end{array}$ & $264.6^{\mathrm{c}}$ \\
\hline Dynamic & & & & & & & & & \\
\hline $\begin{array}{l}\text { Power }(\mathrm{nW}) \\
\text { Total }\end{array}$ & 234.8 & 113.4 & 334.7 & 219.5 & 98.53 & 291.8 & 303.2 & 141.8 & 373.5 \\
\hline $\begin{array}{l}\text { Power (nW) } \\
\text { Worst case }\end{array}$ & $555.95^{\mathrm{d}}$ & $425.75^{\mathrm{d}}$ & $566.5^{\mathrm{d}}$ & $494.1^{\mathrm{d}}$ & $383.88^{\mathrm{d}}$ & $532^{d}$ & $627.6^{\mathrm{d}}$ & $452.9^{\mathrm{d}}$ & $638.1^{\mathrm{d}}$ \\
\hline Delay (ps) & 91.55 & 89.3 & 72.96 & 70.35 & 67.52 & 74.03 & 75.48 & 74.74 & 74.04 \\
\hline $\mathrm{PDP}(\mathrm{aJ})$ & 50.89 & 38.01 & 41.33 & 34.75 & 25.91 & 39.38 & 47.37 & 33.84 & 47.24 \\
\hline Device & $12 \mathrm{MOS}$ & $11 \mathrm{MOS}$ & 19MOS & $12 \mathrm{MOS}$ & $11 \mathrm{MOS}$ & 19MOS & $13 \mathrm{MOS}$ & $12 \mathrm{MOS}$ & $22 \mathrm{MOS}$ \\
\hline Count & $+2 \mathrm{MTJ}$ & $+2 \mathrm{MTJ}$ & & $+2 \mathrm{MTJ}$ & $+2 \mathrm{MTJ}$ & & $+2 \mathrm{MTJ}$ & $+2 \mathrm{MTJ}$ & \\
\hline
\end{tabular}

Write circuit has been excluded in the tabulated values. Static, dynamic, and total power dissipation are the average values for all the input combinations.

${ }^{\mathrm{a}} \mathrm{HG} 1$ and $\mathrm{HG} 2$ are nonvolatile in nature, and thus the supply can be turned off in standby mode

${ }^{\mathrm{b}, \mathrm{c}}$ Static power dissipation in steady state condition

${ }^{c}$ Due to volatile nature of DPTL-C ${ }^{2}$ MOS logic, the power is not allowed to switch off

${ }^{\mathrm{d}}$ Total power dissipation $=$ static power + dynamic power in active mode

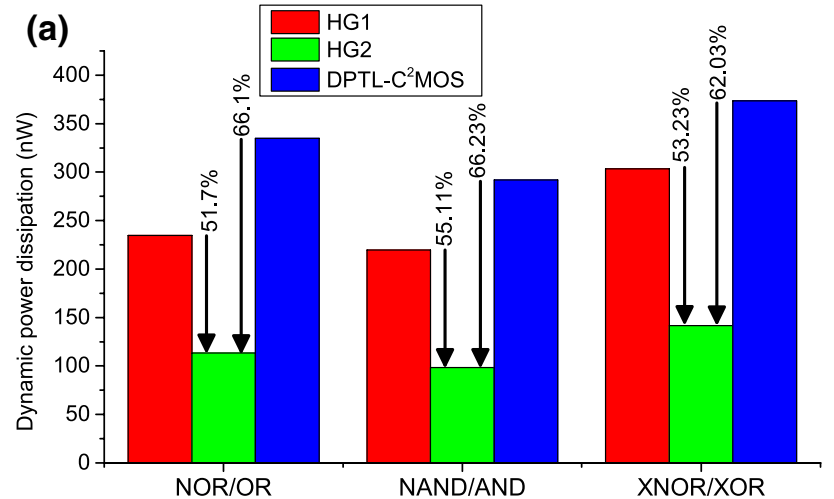

(b)

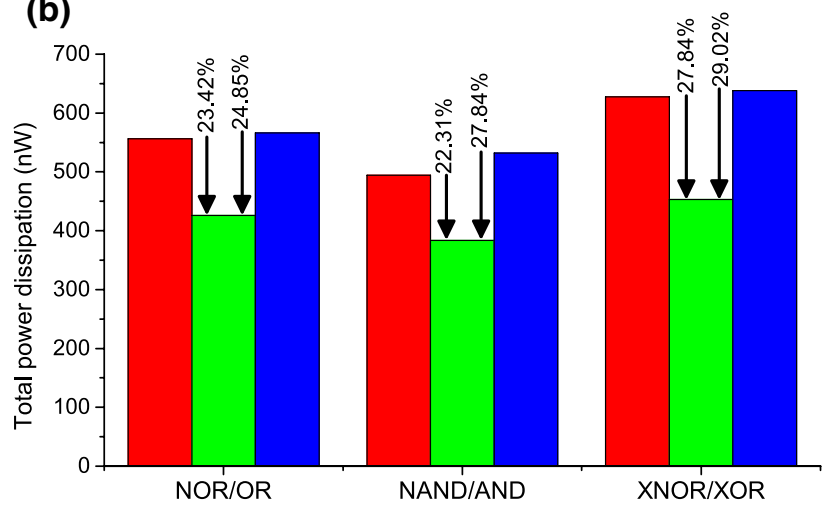

Fig. 10 Comparison of $\mathbf{a}$ the dynamic power dissipation and $\mathbf{b}$ total power dissipation among HG1, HG2 and DPTL-C ${ }^{2}$ MOS logic gates the pull-down transistor N3 of the hybrid gates needs to provide a discharge path for the current (either I1 or I2) due to which the output delay depends upon the size of this transistor. By increasing the width of the N3 transistor the delay of the hybrid gates can be reduced. However, by doing so the power dissipation in the HG2 will increase, which is shown in Table 6 and Fig. 11. Hence, we need to tackle this delay versus power dissipation trade-off for the hybrid gates.

In terms of device count, the HG2 NOR/OR and NAND/ AND gates need only 11 MOS transistors, on the contrary, HG1, and DPTL-C ${ }^{2}$ MOS require 12 and 19 MOS transistors, respectively. Similarly, for XNOR/XOR gate, HG1 and DPTL-C ${ }^{2}$ MOS require 13 and 22 MOS transistors, whereas, for the HG2 XNOR/ XOR gate, only 12 MOS transistors are sufficient. Hence, in terms of the number of transistors HG2 logic gates are superior to HG1 and DPTL-C ${ }^{2}$ MOS logic gates.

The process and mismatch variations during nano-scale fabrication of VLSI circuits affect its performance. To study this effect at the design stage, we have performed MC simulation of 200 runs for the design. During MC simulation, we have not only incorporated CMOS variations, but also, $3 \%$ variations in the TMR, $t_{s l}$ and $t_{o x}$ (refer Table 3 ) that follow Gaussian distribution for the MTJs. Table 7 shows the total power dissipation comparison among all the logic gates. It suggests that HG2 logic gate design dissipates the least power compared to the rest of the designs. 


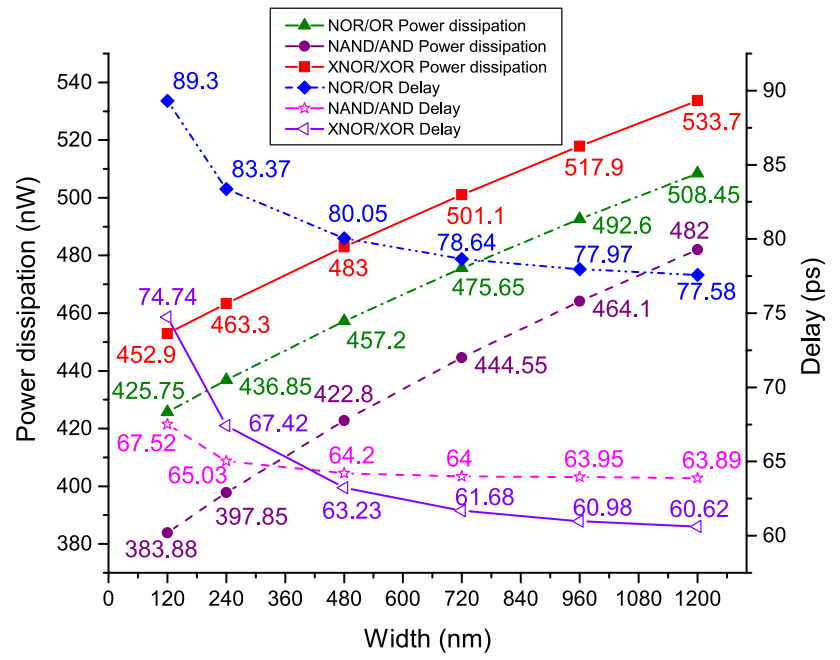

Fig. 11 Dependency plot for power dissipation and delay on the width of the pull-down transistor N3. As N3's width increases, the delay decreases while on the contrary, power dissipation increases

\section{Conclusion}

The major contribution of this work is to provide an in-depth analysis of basic hybrid circuits developed using CMOS logic and p-MTJ device. In this paper, we have designed and presented a detailed analysis of all the logic gates, i.e., NOR/OR, NAND/AND, and XNOR/XOR based on the hybrid SHE+STT-MTJ/CMOS LIM structure. The simulation results obtained substantiates the fact that hybrid logic gates are not only nonvolatile in nature but also are superior to their CMOS counterparts in terms of power dissipation and the number of transistors used in the design. Due to the nonvolatility nature of hybrid logic gates, they can be completely turned off in standby mode to save a significant amount of power without the necessity of backup or restoring process as in the conventional CMOS technology. Hence these circuits can be considered for future low power applications.

\section{Appendix: DPTL-C ${ }^{2}$ MOS-based logic gates}

See Fig. 12.
Table 6 Variation in total power dissipation and delay w.r.t the width of pull-down transistor N3 for HG2

\begin{tabular}{llllllll}
\hline Gate type & N3 Width (nm) & 120 & 240 & 480 & 720 & 960 & 1200 \\
\hline \multirow{2}{*}{ NOR/OR } & Power (nW) & 425.75 & 436.85 & 457.2 & 475.65 & 492.6 & 508.45 \\
& Delay (ps) & 89.3 & 83.37 & 80.05 & 78.64 & 77.97 & 77.58 \\
\multirow{2}{*}{ NAND/AND } & Power (nW) & 383.88 & 397.85 & 422.8 & 444.55 & 464.1 & 482 \\
& Delay (ps) & 67.52 & 65.03 & 64.2 & 64 & 63.95 & 63.89 \\
\multirow{2}{*}{ XNOR/XOR } & Power (nW) & 452.9 & 463.3 & 483 & 501.1 & 517.9 & 533.7 \\
\hline
\end{tabular}

Table 7 Total power dissipation comparison between HG1, HG2 and DPTL- $\mathrm{C}^{2} \mathrm{MO}$ logic gates for MC simulation

\begin{tabular}{lllllll}
\hline Design type & Gate type & $\begin{array}{l}\text { Min } \\
(\mathrm{nW})\end{array}$ & $\begin{array}{l}\text { Max } \\
(\mathrm{nW})\end{array}$ & $\begin{array}{l}\text { Mean } \\
(\mathrm{nW})\end{array}$ & $\begin{array}{l}\text { Median } \\
(\mathrm{nW})\end{array}$ & $\begin{array}{l}\text { SD } \\
(\mathrm{nW})\end{array}$ \\
\hline HG1 & NOR/OR & 515.25 & 596.55 & 554.35 & 554.65 & 13.63 \\
& NAND/AND & 456.6 & 529 & 493.05 & 493.8 & 12.13 \\
& XNOR/XOR & 580.8 & 670.3 & 625.75 & 626.7 & 16.45 \\
HG2 & NOR/OR & 399.35 & 458.6 & 426.9 & 427.35 & 9.72 \\
& NAND/AND & 361 & 407.7 & 384.55 & 384.8 & 7.52 \\
& XNOR/XOR & 422.3 & 496.9 & 455.3 & 454.8 & 13.33 \\
DPTL-C ${ }^{2}$ MOS & NOR/OR & 456.8 & 592.1 & 559.6 & 563.5 & 24.73 \\
& NAND/AND & 501.7 & 562.9 & 532 & 532.7 & 10.6 \\
& XNOR/XOR & 598.9 & 768.1 & 640.3 & 635.2 & 27.42 \\
\hline
\end{tabular}




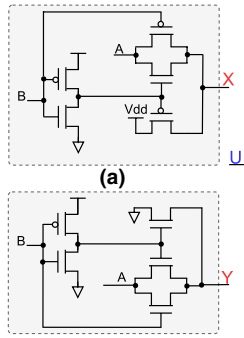

(b)

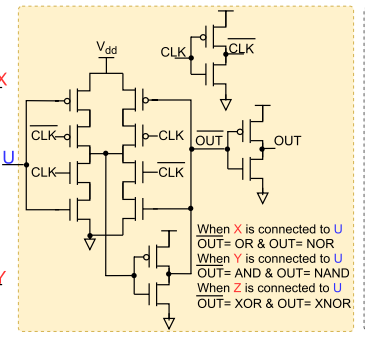

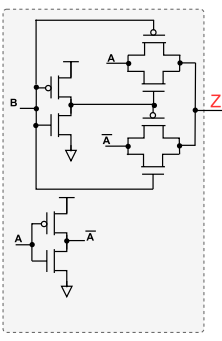

(c)
Fig. 12 DPTL-C ${ }^{2}$ MOS circuit for a NOR/OR gate, b NAND/AND gate, and $\mathbf{c} X N O R / X O R$ gate [9]

Acknowledgements Prashanth Barla would like to acknowledge the Manipal Institute of Technology, Manipal Academy of Higher Education, Manipal, for providing TMA Pai scholarship for his research work. Part of the work presented in Introduction and Background sections has been already reported and refs. $[1,2,9]$ and can be used for detailed understanding.

Funding Open access funding provided by Manipal Academy of Higher Education, Manipal.

Availability of data and material Not applicable.

\section{Declarations}

Conflict of interest The authors declare that they have no conflict of interest.

\section{Code availability Not applicable}

Open Access This article is licensed under a Creative Commons Attribution 4.0 International License, which permits use, sharing, adaptation, distribution and reproduction in any medium or format, as long as you give appropriate credit to the original author(s) and the source, provide a link to the Creative Commons licence, and indicate if changes were made. The images or other third party material in this article are included in the article's Creative Commons licence, unless indicated otherwise in a credit line to the material. If material is not included in the article's Creative Commons licence and your intended use is not permitted by statutory regulation or exceeds the permitted use, you will need to obtain permission directly from the copyright holder. To view a copy of this licence, visit http://creativecommons.org/licenses/by/4.0/.

\section{References}

1. Joshi, V.K., Barla, P., Bhat, S., Kaushik, B.K.: From MTJ device to hybrid CMOS/MTJ circuits: a review. IEEE Access 8, 194105-194146 (2020). https://doi.org/10.1109/ACCESS. 2020.3033023

2. Barla, P., Joshi, V.K., Bhat, S.: Spintronic devices: a promising alternative to CMOS devices. J. Comput. Electron. 20(2), 805-837 (2021). https://doi.org/10.1007/s10825-020-01648-6

3. Sousa, R.C., Prejbeanu, I.L.: Non-volatile magnetic random access memories (MRAM). C. R. Phys. 9(6), 1013-1021 (2005). https://doi.org/10.1016/j.crhy.2005.10.007
4. Zhao, W., Belhaire, E., Chappert, C., Jacquet, F., Mazoyer, P.: New non-volatile logic based on spin-MTJ. Phys. Status Solidi A 205(6), 1373-1377 (2008). https://doi.org/10.1002/pssa. 200778135

5. Chabi, D., Zhao, W., Deng, E., Zhang, Y., Romdhane, N.B., Klein, J.-O., Chappert, C.: Ultra low power magnetic flip-flop based on checkpointing/power gating and self-enable mechanisms. IEEE Trans. Circuits Syst. I Regul. Pap. 61(6), 17551765 (2014). https://doi.org/10.1109/TCSI.2013.2295026

6. Roohi, A., DeMara, R.F.: NV-clustering: normally-off computing using non-volatile datapaths. IEEE Trans. Comput. 67(7), 949-959 (2018). https://doi.org/10.1109/TC.2018.2795601

7. Jaiswal, A., Andrawis, R., Roy, K.: Area-efficient nonvolatile flip-flop based on spin hall effect. IEEE Magn. Lett. 9, 1-4 (2018). https://doi.org/10.1109/LMAG.2018.2829676

8. Kang, W., Ran, Y., Lv, W., Zhang, Y., Zhao, W.: High-speed, low-power, magnetic non-volatile flip-flop with voltage-controlled, magnetic anisotropy assistance. IEEE Magn. Lett. 7, 1-5 (2016). https://doi.org/10.1109/LMAG.2016.2604205

9. Barla, P., Shet, D., Joshi, V. K., Bhat, S.: Design and analysis of LIM hybrid MTJ/CMOS logic gates. In: 2020 5th International Conference on Devices, Circuits and Systems (ICDCS), 2020, pp. 41-45. https://doi.org/10.1109/ICDCS48716.2020.243544

10. Barla, P., Joshi, V.K., Bhat, S.: A novel low power and reduced transistor count magnetic arithmetic logic unit using hybrid STT-MTJ/CMOS circuit. IEEE Access 8, 6876-6889 (2020). https://doi.org/10.1109/ACCESS.2019.2963727

11. Matsunaga, S., Hayakawa, J., Ikeda, S., Miura, K., Endoh, T., Ohno, H., Hanyu, T.: MTJ-based nonvolatile logic-in-memory circuit, future prospects and issues. In: Proceedings of Conference Design, Autom. Test Europe, 2009, pp. 433-435. https:// doi.org/10.1109/DATE.2009.5090704

12. Deng, E., Zhang, Y., Klein, J.-O., Ravelsona, D., Chappert, C., Zhao, W.: Low power magnetic full-adder based on spin transfer torque MRAM. IEEE Trans. Magn. 49(9), 4982-4987 (2013). https://doi.org/10.1109/TMAG.2013.2245911

13. Roohi, A., Zand, R., DeMara, R.F.: A tunable majority gatebased full adder using current-induced domain wall nanomagnets. IEEE Trans. Magn. 52(8), 1-7 (2016). https://doi.org/10. 1109/TMAG.2016.2540600

14. Roohi, A., Zand, R., Fan, D., DeMara, R.F.: Voltage-based concatenatable full adder using spin hall effect switching. IEEE Trans. Comput.-Aid. Des. Integr. Circuits Syst. 36(12), 2134-2138 (2017). https://doi.org/10.1109/TCAD.2017.2661800

15. Zarei, A., Safaei, F.: Power and area-efficient design of VCMAMRAM based full-adder using approximate computing for IoT applications. Microelectron. J. 82, 62-70 (2018). https://doi.org/ 10.1016/j.mejo.2018.10.010

16. An, Q., Su, L., Klein, J.-O., Beux, S.L., OConnor, I., Zhao, W. Full-adder circuit design based on all-spin logic device, 163-168 (2015). https://doi.org/10.1109/NANOARCH.2015.7180606

17. Patra, M., Maiti, S.K.: All-spin logic operations: memory device and reconfigurable computing. EPL 121(3), 38004 (2018). https:// doi.org/10.1209/0295-5075/121/38004

18. An, Q., Beux, S.L., OConnor, I., Klein, J.O., Zhao, W.: Arithmetic Logic Unit based on all-spin logic devices. IEEE (2017). https:// doi.org/10.1109/NEWCAS.2017.8010169

19. Cardoso, S., Leitao, D.C., Gameiro, L., Cardoso, F., Ferreira, R., Paz, E., Freitas, P.P.: Magnetic tunnel junction sensors with pTesla sensitivity. Microsyst. Technol. 20(4), 793-802 (2014). https:// doi.org/10.1007/s00542-013-2035-1

20. Roy, K., Sengupta, A., Shim, Y.: Perspective: stochastic magnetic devices for cognitive computing. J. Appl. Phys. 123(21), 210901 (2018). https://doi.org/10.1063/1.5020168

21. Wijesinghe, P.: Neuro-inspired computing enhanced by scalable algorithms and physics of emerging nanoscale resistive devices, 
Ph.D. thesis, Purdue University Graduate School (2019). https:// doi.org/10.25394/PGS.8273483.v1

22. Lin, X., Yang, W., Wang, K.L., Zhao, W.: Two-dimensional spintronics for low-power electronics. Nat. Electron. 2(7), 274-283 (2019). https://doi.org/10.1038/s41928-019-0273-7

23. Jinnai, B., Watanabe, K., Fukami, S., Ohno, H.: Scaling magnetic tunnel junction down to single-digit nanometers-Challenges and prospects. Appl. Phys. Lett. 116(16), 160501 (2020). https://doi. org/10.1063/5.0004434

24. Wang, Z., Zhao, W., Kang, W., Bouchenak-Khelladi, A., Zhang, Y., Zhang, Y., Klein, J.-O., Ravelosona, D., Chappert, C.: A physics-based compact model of ferroelectric tunnel junction for memory and logic design. J. Phys. D Appl. Phys. 47(4), 045001 (2013). https://doi.org/10.1088/0022-3727/47/4/045001

25. Luo, Z., Hrabec, A., Dao, T.P., Sala, G., Finizio, S., Feng, J., Mayr, S., Raabe, J., Gambardella, P., Heyderman, L.J.: Currentdriven magnetic domain-wall logic. Nature 579(7798), 214-218 (2020). https://doi.org/10.1038/s41586-020-2061-y

26. Mankalale, M.G., Zhao, Z., Wang, J.-P., Sapatnekar, S.S.: SkyLogic-a proposal for a skyrmion-based logic device. IEEE Trans. Electron Devices 66(4), 1990-1996 (2019). https://doi.org/10. 1109/TED.2019.2899263

27. Zhang, Z., Zhu, Y., Zhang, Y., Zhang, K., Nan, J., Zheng, Z., Zhang, Y., Zhao, W.: Skyrmion-based ultra-low power electricfield-controlled reconfigurable (SUPER) logic gate. IEEE Electron Device Lett. 40(12), 1984-1987 (2019). https://doi.org/10. 1109/LED.2019.2946863

28. Behin-Aein, B., Datta, D., Salahuddin, S., Datta, S.: Proposal for an all-spin logic device with built-in memory. Nat. Nanotechnol. 5(4), 266-270 (2010). https://doi.org/10.1038/nnano.2010.31

29. Zhao, W., Prenat, G.: Spintronics-Based Computing. Springer, Berlin (2015). https://doi.org/10.1007/978-3-319-15180-9

30. Kang, W., Deng, E., Wang, Z., Zhao, W.: Spintronic Logic-inMemory Paradigms and Implementations. In: Suri M. (eds) Applications of Emerging Memory Technology., Springer Series in Advanced Microelectronics 63 (2020) 215-229. https://doi.org/ 10.1007/978-981-13-8379-3-9

31. Slonczewski, J.C.: Current-driven excitation of magnetic multilayers. J. Magn. Magn. Mater. 159(1), L1-L7 (1996). https://doi.org/ 10.1016/0304-8853(96)00062-5

32. Berger, L.: Emission of spin waves by a magnetic multilayer traversed by a current. Phys. Rev. B 54(13), 9353-9358 (1996). https://doi.org/10.1103/PhysRevB.54.9353

33. Huai, Y., Albert, F., Nguyen, P., Pakala, M., Valet, T.: Observation of spin-transfer switching in deep submicron-sized and lowresistance magnetic tunnel junctions. Appl. Phys. Lett. 84(16), 3118-3120 (2004). https://doi.org/10.1063/1.1707228

34. DYakonov, M. I., Perel, V. I.:https://ui.adsabs.harvard.edu/abs/ 1971JETPL..13..467D/abstractPossibility of Orienting Electron Spins with Current, JETPL 13 (1971) 467

35. Hirsch, J.E.: Spin hall effect. Phys. Rev. Lett. 83(9), 1834-1837 (1999). https://doi.org/10.1103/PhysRevLett.83.1834

36. Saitoh, E., Ueda, M., Miyajima, H., Tatara, G.: Conversion of spin current into charge current at room temperature: Inverse spin-Hall effect. Appl. Phys. Lett. 88(18), 182509 (2006). https://doi.org/10. $1063 / 1.2199473$

37. Wolf, S.A., Awschalom, D.D., Buhrman, R.A., Daughton, J.M., von Molnár, S., Roukes, M.L., Chtchelkanova, A.Y., Treger, D.M.: Spintronics: a spin-based electronics vision for the future. Science 294(5546), 1488-1495 (2001). https://doi.org/10.1126/science. 1065389

38. Shiota, Y., Nozaki, T., Bonell, F., Murakami, S., Shinjo, T., Suzuki, Y.: Induction of coherent magnetization switching in a few atomic layers of FeCo using voltage pulses. Nat. Mater. 11(1), 39-43 (2012). https://doi.org/10.1038/nmat3172
39. Chu, Y.-H., Martin, L.W., Holcomb, M.B., Gajek, M., Han, S.-J., He, Q., Balke, N., Yang, C.-H., Lee, D., Hu, W., Zhan, Q., Yang, P.-L., Fraile-Rodríguez, A., Scholl, A., Wang, S.X., Ramesh, R.: Electric-field control of local ferromagnetism using a magnetoelectric multiferroic. Nat. Mater. 7(6), 478-482 (2008). https:// doi.org/10.1038/nmat2184

40. Nozaki, T., Shiota, Y., Shiraishi, M., Shinjo, T., Suzuki, Y.: Voltage-induced perpendicular magnetic anisotropy change in magnetic tunnel junctions. Appl. Phys. Lett. 96(2), 022506 (2010). https://doi.org/10.1063/1.3279157

41. Song, Y.J., Lee, J.H., Shin, H.C., Lee, K.H., Suh, K., Kang, J.R., Pyo, S.S., Jung, H.T., Hwang, S.H., Koh, G.H., Oh, S.C., Park, S.O., Kim, J.K., Park, J.C., Kim, J., Hwang, K.H., Jeong, G.T., Lee, K.P., Jung, E.S.:Highly functional and reliable 8Mb STTMRAM embedded in 28nm logic, 2016 IEEE International Electron Devices Meeting (IEDM) (2016) 27.2.1-27.2.4 https://doi. org/10.1109/IEDM.2016.7838491

42. Chung, S.-W., Kishi, T., Park, J.W., Yoshikawa, M., Park, K.S., Nagase, T., Sunouchi, K., Kanaya, H., Kim, G.C., Noma, K., Lee, M.S., Yamamoto, A., Rho, K.M., Tsuchida, K., Chung, S.J., Yi, J.Y., Kim, H.S., Chun, Y.S., Oyamatsu, H., Hong, S.J.:4Gbit density STT-MRAM using perpendicular MTJ realized with compact cell structure, 2016 IEEE International Electron Devices Meeting (IEDM) (2016) 27.1.1-27.1.4 https://doi.org/10.1109/IEDM. 2016.7838490

43. Lu, Y., Zhong, T., Hsu, W., Kim, S., Lu, X., Kan, J.J., Park, C., Chen, W.C., Li, X., Zhu, X., Wang, P., Gottwald, M., Fatehi, J., Seward, L., Kim, J.P., Yu, N., Jan, G., Haq, J., Le, S., Wang, Y.J., Thomas, L., Zhu, J., Liu, H., Lee, Y.J., Tong, R.Y., Pi, K., Shen, D., He, R., Teng, Z., Lam, V., Annapragada, R., Torng, T., Wang, P.-K., Kang, S.H.: Fully functional perpendicular STT-MRAM macro embedded in $40 \mathrm{~nm}$ logic for energy-efficient IOT applications, 2015 IEEE International Electron Devices Meeting (IEDM) (2015) 26.1.1-26.1.4 https://doi.org/10.1109/IEDM.2015.74097 70

44. https://www.everspin.com/spin-transfer-torque-mram-produ ctsSpin-transfer Torque MRAM Products Everspin (2021)

45. Yoda, H., Fujita, S., Shimomura, N., Kitagawa, E., Abe, K., Nomura, K., Noguchi, H., Ito, J.:Progress of STT-MRAM technology and the effect on normally-off computing systems. In: 2012 International Electron Devices Meeting, 2012, pp. 11.3.1-11.3.4. https://doi.org/10.1109/IEDM.2012.6479023

46. Endoh, T., Koike, H., Ikeda, S., Hanyu, T., Ohno, H.: An overview of nonvolatile emerging memories- spintronics for working memories. IEEE J. Emerg. Sel. Top. Circuits Syst. 6(2), 109-119 (2016). https://doi.org/10.1109/JETCAS.2016.2547704

47. Kang, W., Zhao, W., Deng, E., Klein, J.-O., Cheng, Y., Ravelosona, D., Zhang, Y., Chappert, C.: A radiation hardened hybrid spintronic/CMOS nonvolatile unit using magnetic tunnel junctions. J. Phys. D Appl. Phys. 47(40), 405003 (2014). https://doi. org/10.1088/0022-3727/47/40/405003

48. Onizawai, N., Hanyu, T.:Soft-error tolerant transistor/ magnetictunnel-junction hybrid non-volatile C-element. IEICE Electron. Express (2014) 11.20141017 https://doi.org/10.1587/elex.11. 20141017

49. Wang, Z., Zhou, H., Wang, M., Cai, W., Zhu, D., Klein, J.-O., Zhao, W.: Proposal of toggle spin torques magnetic RAM for ultrafast computing. IEEE Electron Device Lett. 40(5), 726-729 (2019). https://doi.org/10.1109/LED.2019.2907063

50. Mangin, S., Ravelosona, D., Katine, J.A., Carey, M.J., Terris, B.D., Fullerton, E.E.: Current-induced magnetization reversal in nanopillars with perpendicular anisotropy. Nat. Mater. 5, 210-215 (2006). https://doi.org/10.1038/nmat1595

51. Kishi, T., Yoda, H., Kai, T., Nagase, T., Kitagawa, E., Yoshikawa, M., Nishiyama, K., Daibou, T., Nagamine, M., Amano, 
M., Takahashi, S., Nakayama, M., Shimomura, N., Aikawa, H., Ikegawa, S., Yuasa, S., Yakushiji, K., Kubota, H., Fukushima, A., Oogane, M., Miyazaki, T., Ando, K.: Lower-current and fast switching of a perpendicular TMR for high speed and high density spin-transfer-torque MRAM. https://doi.org/10.1109/IEDM.2008. 4796680

52. Yoshikawa, M., Kitagawa, E., Nagase, T., Daibou, T., Nagamine, M., Nishiyama, K., Kishi, T., Yoda, H.: Tunnel magnetoresistance over $100 \%$ in MgO-based magnetic tunnel junction films with perpendicular magnetic L1_\{0\}-FePt electrodes. IEEE Trans. Magn. 44(11), 2573-2576 (2008). https://doi.org/10.1109/TMAG.2008. 2003059

53. Ikeda, S., Miura, K., Yamamoto, H., Mizunuma, K., Gan, H.D., Endo, M., Kanai, S., Hayakawa, J., Matsukura, F., Ohno, H.: A perpendicular-anisotropy $\mathrm{CoFeB}-\mathrm{MgO}$ magnetic tunnel junction. Nat. Mater. 9(9), 721-724 (2010). https://doi.org/10.1038/nmat2 804

54. Barla, P., Joshi, V.K., Bhat, S.: A novel self write-terminated driver for hybrid STT-MTJ/CMOS LIM structure. Ain Shams Eng. J. 12(2), 1839-1847 (2021). https://doi.org/10.1016/j.asej. 2020.10.012

55. Mihai Miron, I., Gaudin, G., Auffret, S., Rodmacq, B., Schuhl, A., Pizzini, S., Vogel, J., Gambardella, P.: Current-driven spin torque induced by the Rashba effect in a ferromagnetic metal layer. Nat. Mater. 9(3), 230-234 (2010)

56. Miron, I.M., Garello, K., Gaudin, G., Zermatten, P.-J., Costache, M.V., Auffret, S., Bandiera, S., Rodmacq, B., Schuhl, A., Gambardella, P.: Perpendicular switching of a single ferromagnetic layer induced by in-plane current injection. Nature 476, 189-193 (2011). https://doi.org/10.1038/nature10309

57. Liu, L., Pai, C.-F., Li, Y., Tseng, H.W., Ralph, D.C., Buhrman, R.A.: Spin-torque switching with the giant spin hall effect of tantalum. Science 336(6081), 555-558 (2012). https://doi.org/10. 1126/science. 1218197

58. Bychkov, Y.A., Rashba, É.I.: Properties of a 2 d electron gas with lifted spectral degeneracy. JETP Lett. 39(2), 78 (1984)

59. Zhang, S.: Spin hall effect in the presence of spin diffusion. Phys. Rev. Lett. 85(2), 393-396 (2000). https://doi.org/10.1103/PhysR evLett.85.393

60. Wang, Z., Zhao, W., Deng, E., Klein, J.-O., Chappert, C.: Perpendicular-anisotropy magnetic tunnel junction switched by spinHall-assisted spin-transfer torque. J. Phys. D Appl. Phys. 48(6), 065001 (2015). https://doi.org/10.1088/0022-3727/48/6/065001

61. van den Brink, A., Cosemans, S., Cornelissen, S., Manfrini, M., Vaysset, A., Van Roy, W., Min, T., Swagten, H.J.M., Koopmans, B.: Spin-Hall-assisted magnetic random access memory. Appl. Phys. Lett. 104(1), 012403 (2014). https://doi.org/10.1063/1. 4858465

62. Kang, W., Lv, W., Zhang, Y., Zhao, W.: Low store power highspeed high-density nonvolatile SRAM design with spin hall effectdriven magnetic tunnel junctions. IEEE Trans. Nanotechnol. 16(1), 148-154 (2017). https://doi.org/10.1109/TNANO.2016. 2640338

63. Wang, Z., Zhang, L., Wang, M., Wang, Z., Zhu, D., Zhang, Y., Zhao, W.: High-density NAND-like spin transfer torque memory with spin orbit torque erase operation. IEEE Electron Device Lett. 39(3), 343-346 (2018). https://doi.org/10.1109/LED.2018.27950 39

64. Wang, Z., Zhao, W., Deng, E., Zhang, Y., Klein, J.-O.: Magnetic non-volatile flip-flop with spin-Hall assistance. Phys. Status Solidi RRL 9(6), 375-378 (2015). https://doi.org/10.1002/pssr.20151 0097
65. Javadi, A.A., Morsali, M., Moaiyeri, M.H.: Magnetic nonvolatile flip-flops with spin-Hall assistance for power gating in ternary systems. J. Comput. Electron. 19(3), 1175-1186 (2020). https:// doi.org/10.1007/s10825-020-01516-3

66. Amirany, A., Rajaei, R.: Fully nonvolatile and low power full adder based on spin transfer torque magnetic tunnel junction with spin-hall effect assistance. IEEE Trans. Magn. 54(12), 1-7 (2018). https://doi.org/10.1109/TMAG.2018.2869811

67. Deng, E., Wang, Z., Klein, J.-O., Prenat, G., Dieny, B., Zhao, W.: High-frequency low-power magnetic full-adder based on magnetic tunnel junction with spin-hall assistance. IEEE Trans. Magn. 51(11), 1-4 (2015). https://doi.org/10.1109/TMAG.2015.2449554

68. Parveen, F., Angizi, S., He, Z., Fan, D.: Low power in-memory computing based on dual-mode SOT-MRAM. In: IEEE/ACM International Symposium on Low Power Electronics and Design (ISLPED). IEEE 2017, 1-6 (2017). https://doi.org/10.1109/ ISLPED.2017.8009200

69. Wang, C., Wang, Z., Xu, Y., Yang, J., Zhang, Y., Zhao, W., Based, Computing-in-Memory Architecture., on Field-Free SOT-MRAM with Self-Reference Method, in, : IEEE International Symposium on Circuits and Systems (ISCAS). IEEE 2020, 1-4 (2020). https:// doi.org/10.1109/ISCAS45731.2020.9180668

70. Johnson, M.T., Bloemen, P.J.H., Broeder, F.J.A.D., de Vries, J.J.: Magnetic anisotropy in metallic multilayers. Rep. Prog. Phys. 59(11), 1409-1458 (1996). https://doi.org/10.1088/0034-4885/ $59 / 11 / 002$

71. Draaisma, H.J.G., de Jonge, W.J.M., Den Broeder, F.J.A.: Magnetic interface anisotropy in $\mathrm{Pd} / \mathrm{Co}$ and $\mathrm{Pd} / \mathrm{Fe}$ multilayers. J. Magn. Magn. Mater. 66(3), 351-355 (1987). https://doi.org/10. 1016/0304-8853(87)90169-7

72. Bandyopadhyay, S., Cahay, M.: Introduction to Spintronics. CRC Press, Boca Raton (2015)

73. Francis, M.N., David, B.N.H.: The scattering of fast electrons by atomic nuclei. Proc. R. Soc. Lond. A 124(794), 425-442 (1929). https://doi.org/10.1098/rspa.1929.0127

74. Smit, J.: The spontaneous hall effect in ferromagnetics II. Physica 24(1), 39-51 (1958). https://doi.org/10.1016/S0031-8914(58) 93541-9

75. Kautz, W.H.: Cellular logic-in-memory arrays. IEEE Trans. Comput. C 18(8), 719-727 (1969). https://doi.org/10.1109/T-C.1969. 222754

76. Stone, H.S.: A logic-in-memory computer. IEEE Trans. Comput. C 19(1), 73-78 (1970). https://doi.org/10.1109/TC.1970.5008902

77. Hanyu, T., Endoh, T., Suzuki, D., Koike, H., Ma, Y., Onizawa, N., Natsui, M., Ikeda, S., Ohno, H.: Standby-power-free integrated circuits using MTJ-based VLSI computing. Proc. IEEE 104(10), 1844-1863 (2016). https://doi.org/10.1109/JPROC.2016.2574939

78. Thapliyal, H., Sharifi, F., Kumar, S.D.: Energy-efficient design of hybrid MTJ/CMOS and MTJ/nanoelectronics circuits. IEEE Trans. Magn. 54(7), 1-8 (2018). https://doi.org/10.1109/TMAG. 2018.2833431

79. Zhao, W., Chappert, C., Javerliac, V., Noziere, J.-P.: High speed, high stability and low power sensing amplifier for MTJ/CMOS hybrid logic circuits. IEEE Trans. Magn. 45(10), 3784-3787 (2009). https://doi.org/10.1109/TMAG.2009.2024325

Publisher's Note Springer Nature remains neutral with regard to jurisdictional claims in published maps and institutional affiliations. 